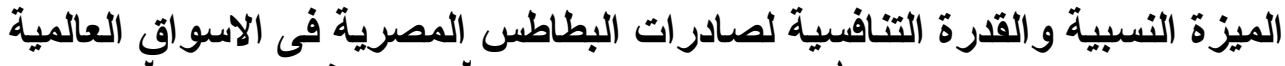

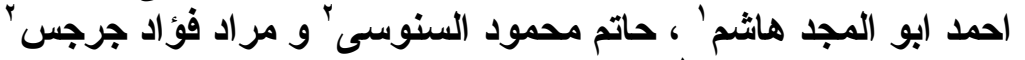

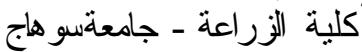

$$
\begin{aligned}
& \text { "معهد بوث الإقصاد الزراعى، هركز البوثث الزراعية }
\end{aligned}
$$

Received on: $15 / 11 / 2017$

Accepted for publication on: 4/12/2017

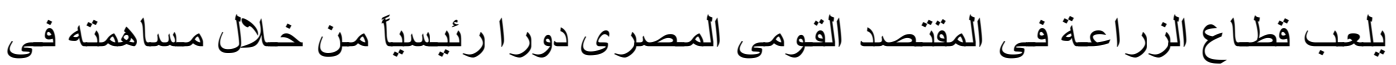

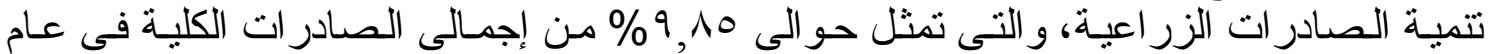

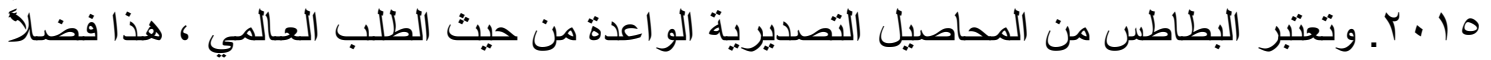

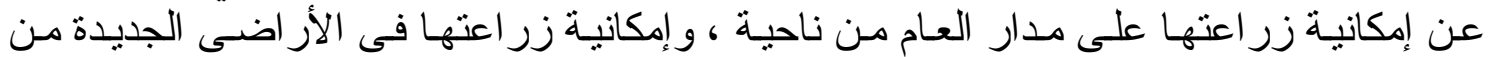

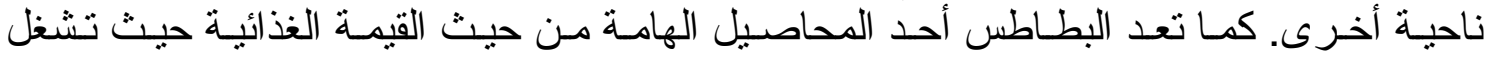

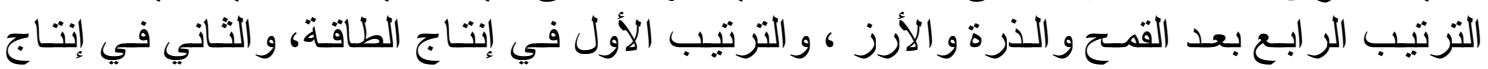

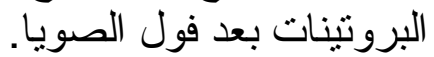

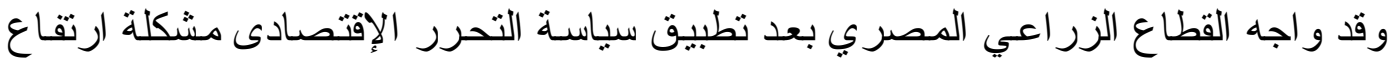

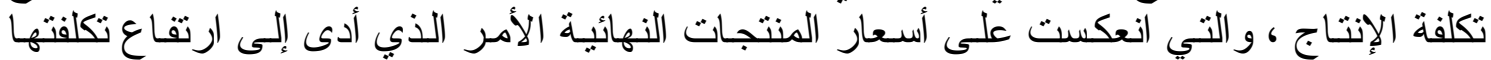

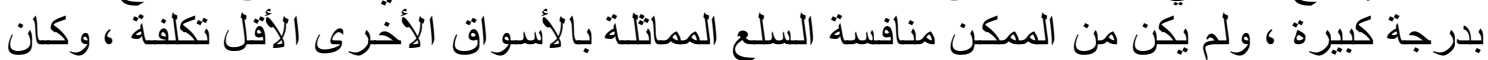

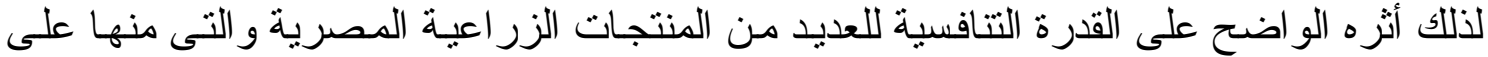
سبيل المثال لا الحصر البطاطس.

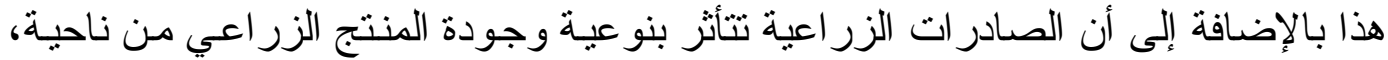

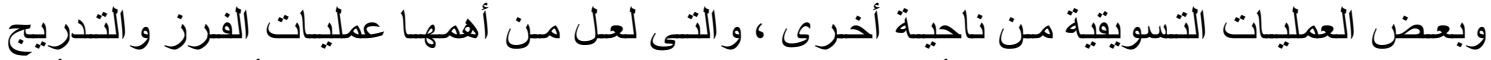

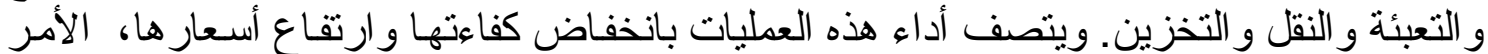

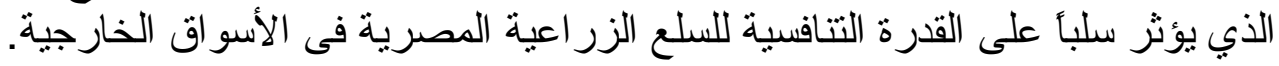

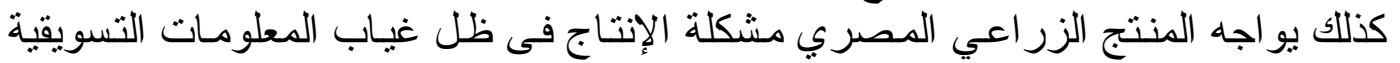

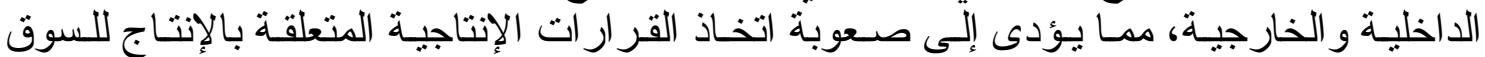

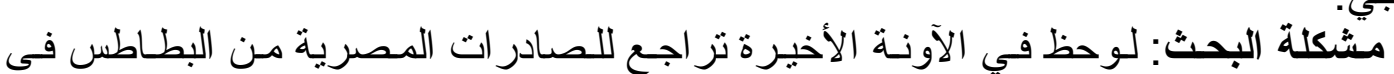

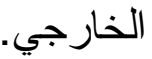

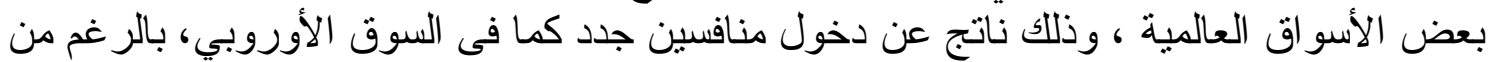

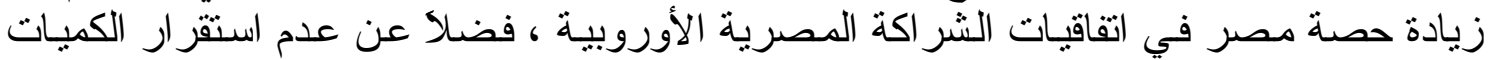

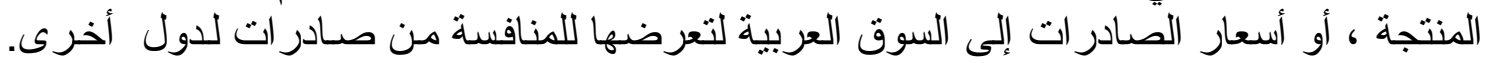

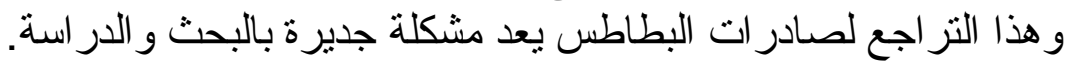

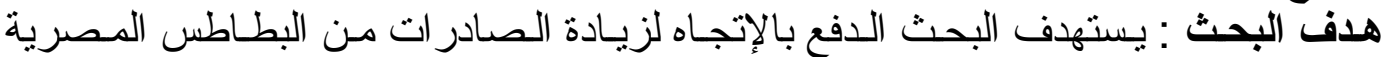

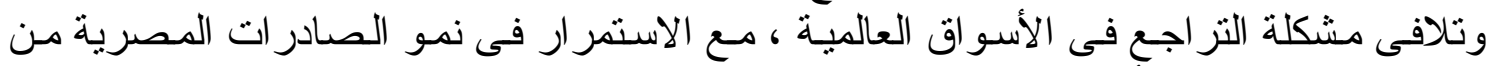

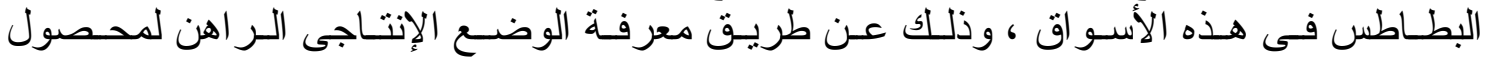

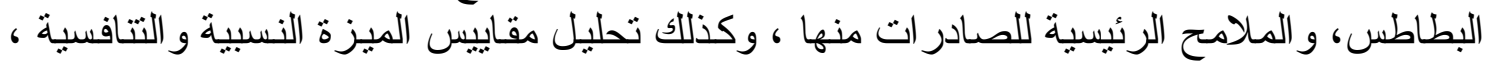

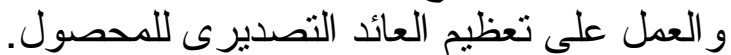

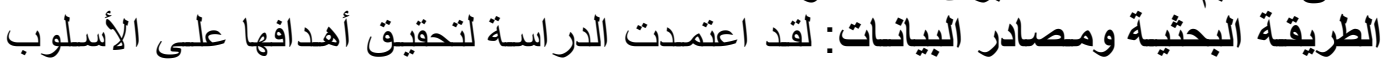

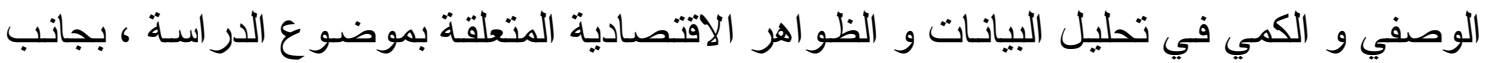

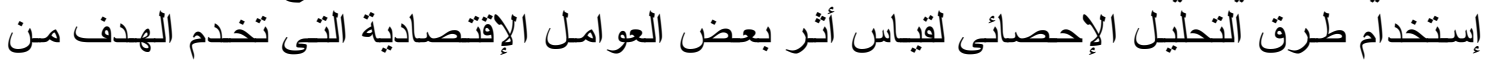

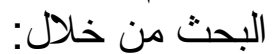

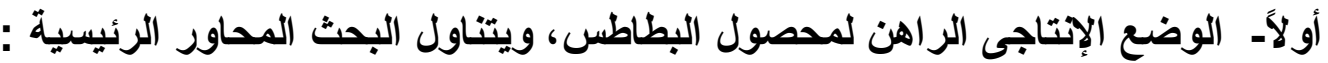

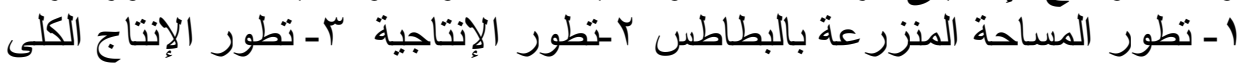




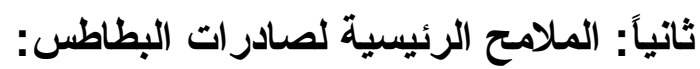

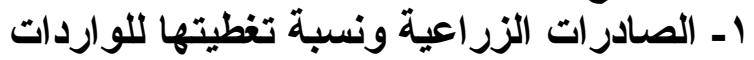
ץ- التركيز الجغرافى للصادرات المصرية من البطاطس لأهم الدول بإستخدام معامل جينى: $\mathrm{Cjx}=100 \sqrt{ }(\mathrm{xsj} / \mathrm{xi})^{2}$ ويتم حسابه كالآتى: التئي

CIX =XSJ XI

أـ السعر النسبى: وهو التهبية بين متوسط أسعار التصدير لأهم الدول المنافسة إلى سعر بـ مؤشر ات الميزة التنافسية : النية PAJ = PC/PE : تصدير الدولة ويحسب كالآتى حيث PAJ : النسبة بين متوسط أسعار الدول المنافسة إلى سعر الدولة المصدرة . متوسط أسعار أهم الدول المنافسة.

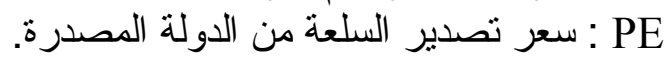
و إذاز ادت قيمة هذا المؤشر عن الو احد الصحيح دل ذلك على لـى أن مصر لها لهيزة لتحافسية

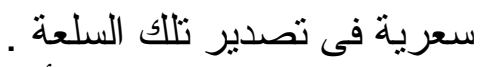
ب- الإتتاج النسبى : أى النسبة بين متوسط إنتاج الدولة من السلعة ومتوسط الإنتاج العـالمى ج- الأهمية النسبية و النصيب السوقى للصادر ات المصرية من البطاطس:

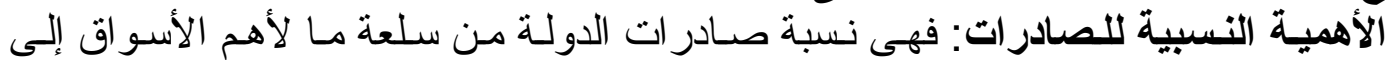

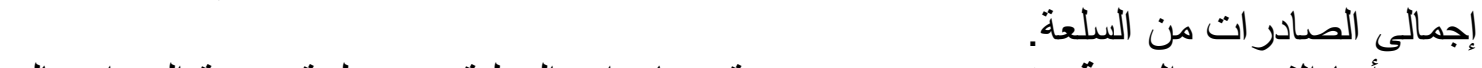

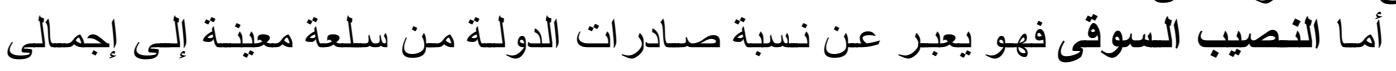
و اردات تلاك السوق للسلعة.

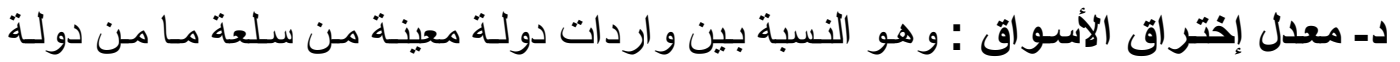

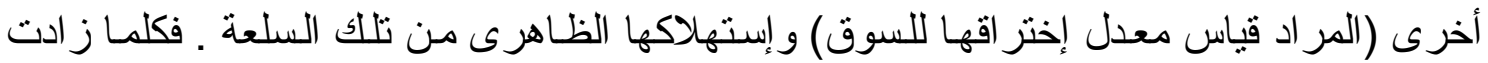

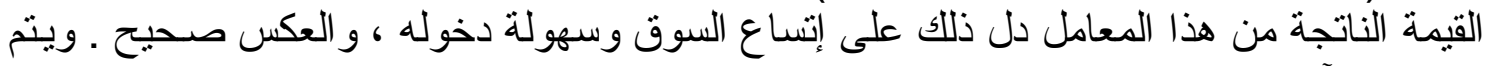

$$
\mathrm{MPRij}=\mathrm{Exij} /(\mathrm{Qij}+\mathrm{Mij}-\mathrm{xij}) 100
$$
حسابه كالآتى :

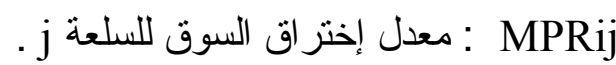

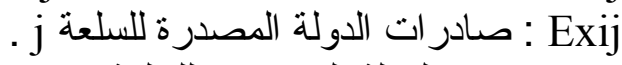

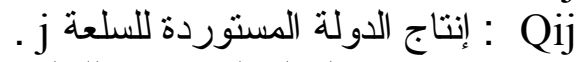

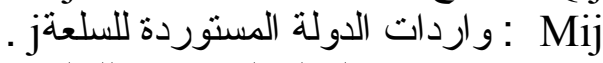

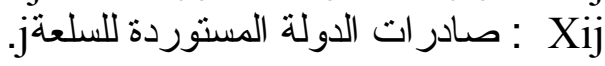

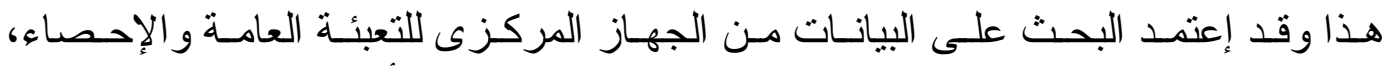

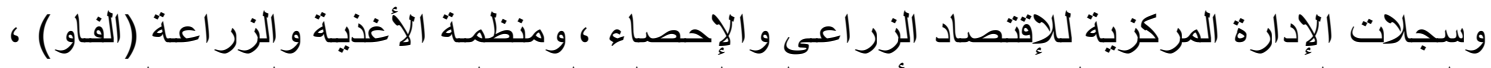
و التقارير المنشورة وغير المنشورة بالأجهزة المختلفة والتى لها صلة بموضورع البحث و الدر اسة. 
ا ـ تطـور المسـاحة المزروعـة بالبطـاطس: إتـضح مـن جـدول رقـم (1) أن المسـاحة

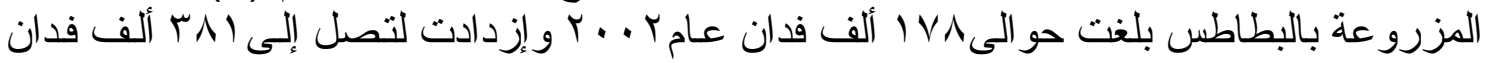

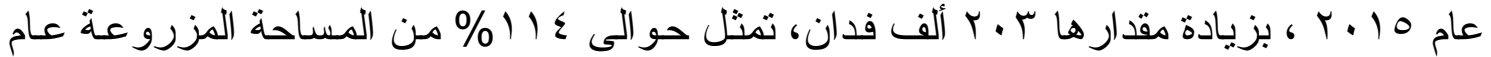

\begin{tabular}{|c|c|c|c|}
\hline (لالانتاج) & (لان/ فذاني) & 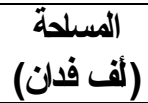 & السنوت \\
\hline IVTO & $9,9)$ & $\mathrm{IVA}$ & $r \ldots r$ \\
\hline 19.4 & $1,, 10$ & $1 \Lambda$. & $r \ldots r$ \\
\hline 196 & $1 ., 0 \mathrm{~V}$ & 197 & $Y \ldots \varepsilon$ \\
\hline T.Tq & $1 ., 1 Y$ & $19 \mathrm{~V}$ & $Y \ldots \theta$ \\
\hline TOSV & ס, 1. & T乡 & $Y \ldots T$ \\
\hline MTV & $1 \cdot, T V$ & r.1 & $r \ldots v$ \\
\hline TM & $1,0 Y$ & $\pi$. & $r \ldots \Lambda$ \\
\hline M. & $1 ., 01$ & Tot & $r \ldots q$ \\
\hline rotV & $1 \cdot, \mathrm{VA}$ & $\pi$ & $r .1$. \\
\hline 1709 & $1 ., 9$. & $M q q$ & $r \cdot 11$ \\
\hline ह צ & $1 \cdot, I Y$ & $\pi \varepsilon$ & $r \cdot I r$ \\
\hline וTו & $1 . M$ & pq. & $r \cdot I r$ \\
\hline EVOA & $11, T V$ & $\sum \pi$ & $Y \cdot 1 \leq$ \\
\hline 2 SMO & 11,19 & HNI & $Y .10$ \\
\hline r.o. & $1 ., 01$ & rAY,VA & المتوسط \\
\hline
\end{tabular}

المصدر: وز ارة الزر اعة و إستصلاح الأر اضى ، الإدارة المركزية للإقتصاد الزر اعى، النشرة الإقتصادية، أعداد منفرقة.

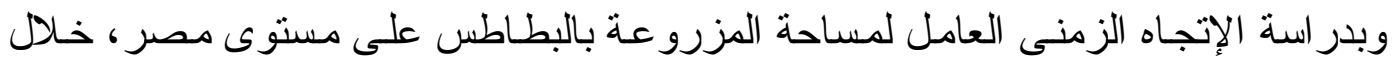

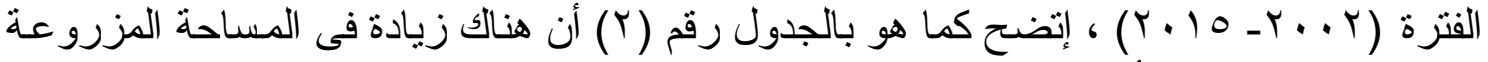

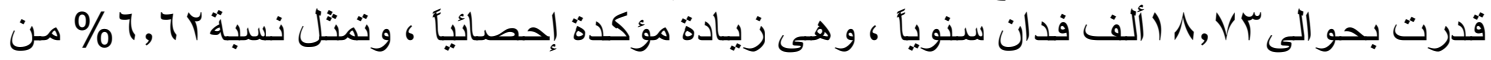

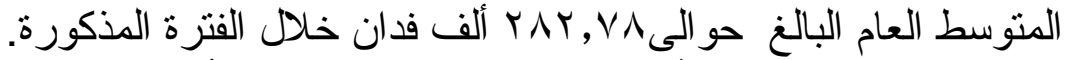

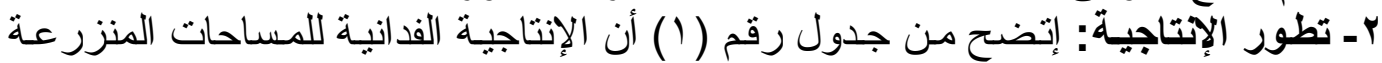

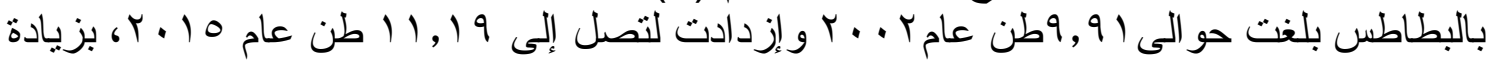

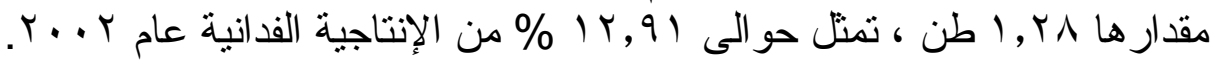


جدول رقم (Y). الإتجـاه الزمنى العام للمؤشرات الانتاجية لمحصول البطاطس فى مصر خلا القترة

\begin{tabular}{|c|c|c|c|c|c|}
\hline \multicolumn{6}{|c|}{$(r+10-r \cdots r)$} \\
\hline التغير & المتوبط & $\mathbf{R}^{2}$ & $\mathbf{F}$ & معادلة الإججاه العام & البيان \\
\hline r & KAT,YA & $\cdot, M$ & $* * 90,1 \leqslant$ & 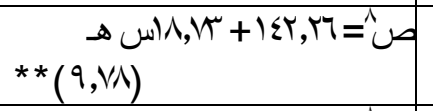 & المسلحة (الهـ فدان) \\
\hline •,07 & $1 ., 01$ & $\cdot, \leqslant \varepsilon$ & $* * 9,7)$ & 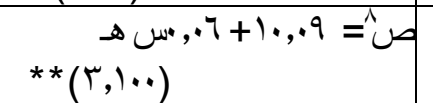 & الإنتاجية ( الطن) \\
\hline$\vee, £ 0$ & r.o. & •,१. & $* * 1,9,71$ & 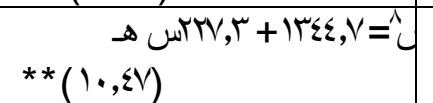 & الإنتاج (الأهـطن) \\
\hline
\end{tabular}

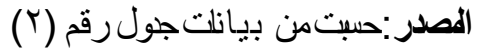

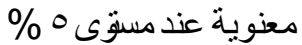

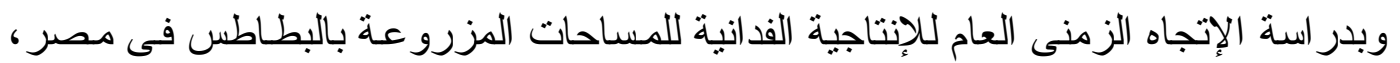

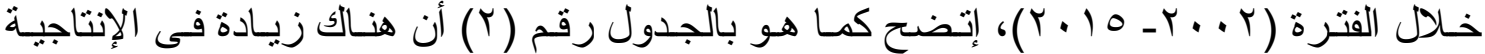

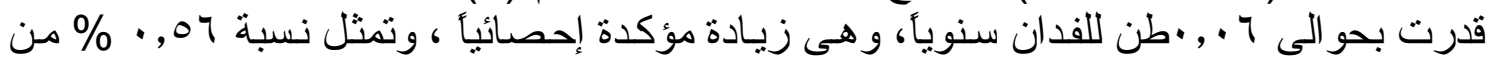

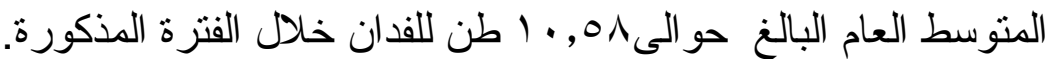

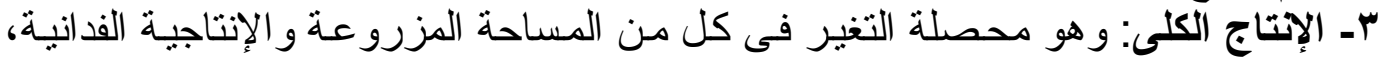

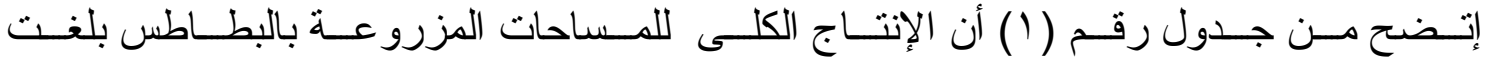

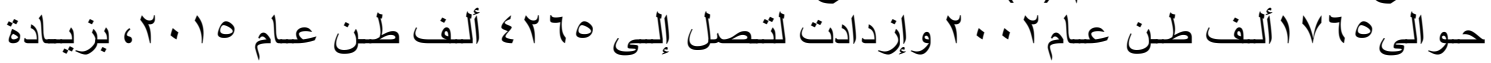

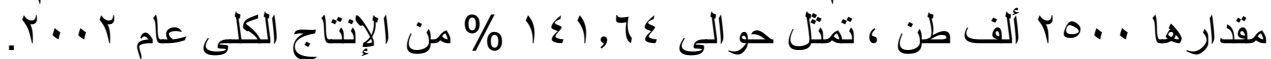

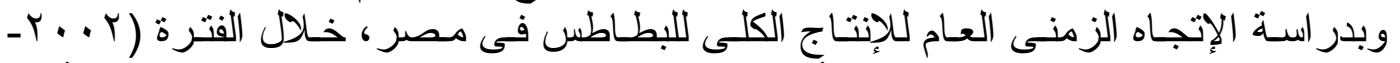
10

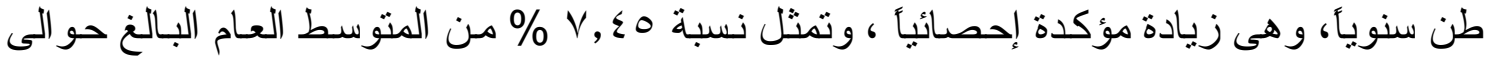

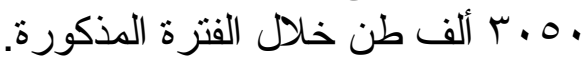

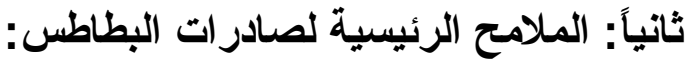

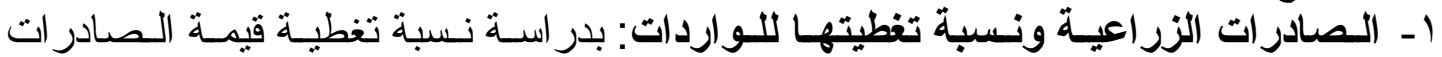

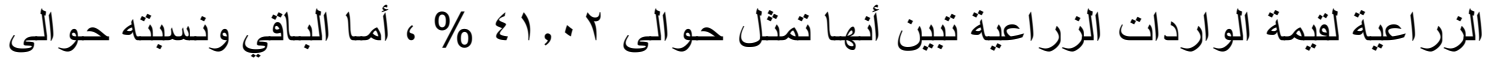

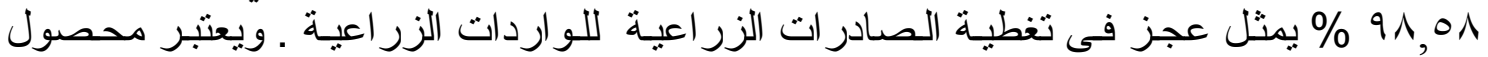

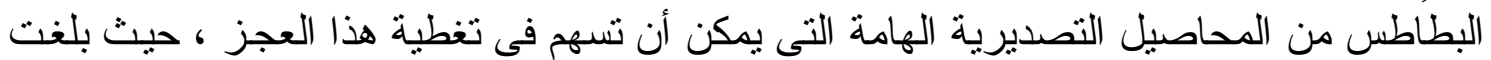

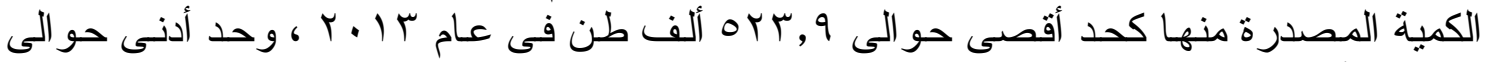

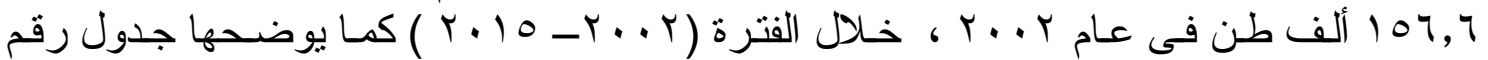

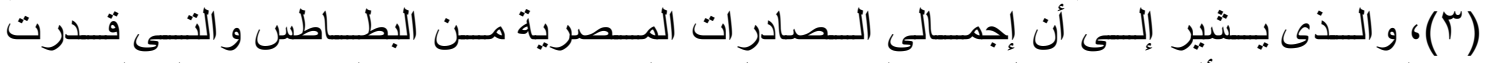

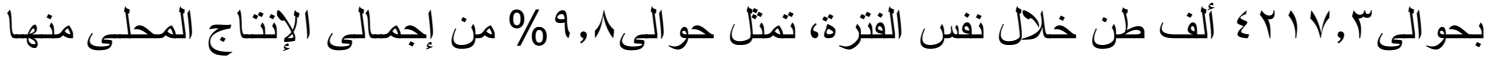

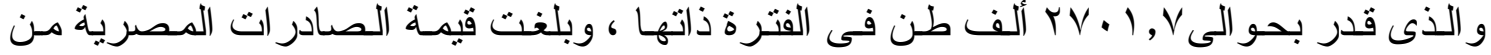

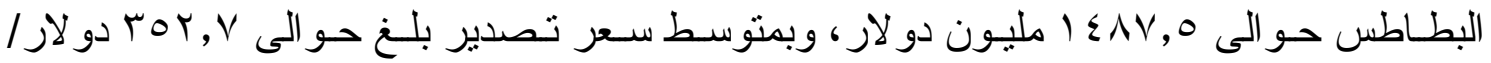
للطن.

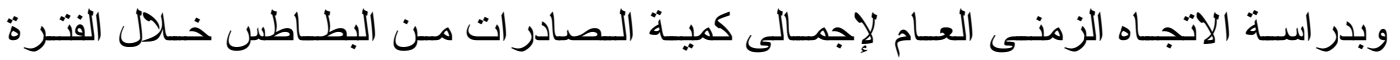

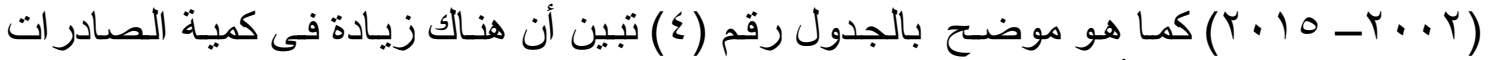

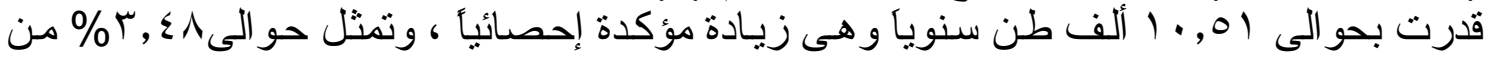

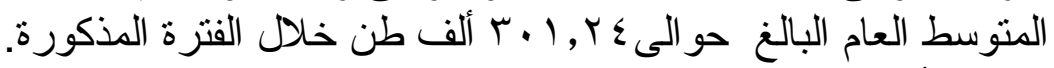

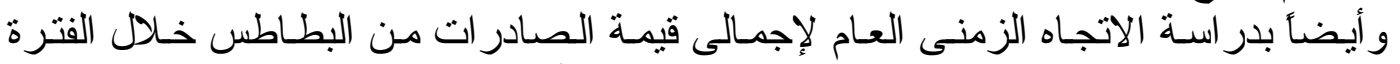

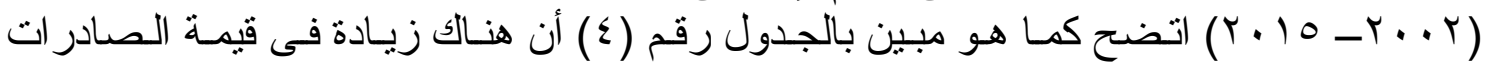




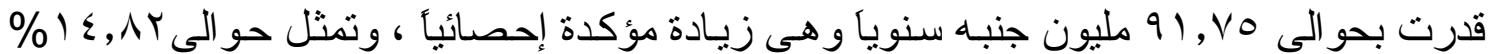

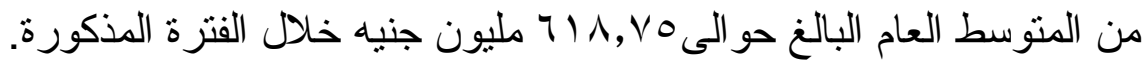

جدول رقم (r). قيمة الصادرات والواردات الكلية و الزراعية ويصض مؤشرات التجارة الخارجية للبطاطس المصرية فى الفترة

\begin{tabular}{|c|c|c|c|c|c|c|c|c|}
\hline & & & & & & & $10=P$. & \\
\hline 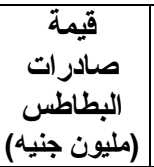 & متوسط سعرف & 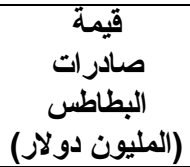 & صادريةت & 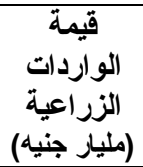 & 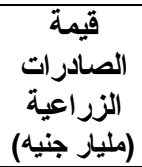 & قيمة الواردات & 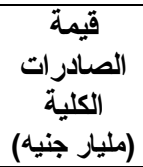 & السنوات \\
\hline $1 \cdot 1, \cdot 1$ & $\Gamma, \Lambda$ & $r\urcorner, 7$ & 107,7 & $7, \varepsilon$ & $1, V$ & $\varepsilon \wedge, 7$ & $17, r$ & $r \ldots r$ \\
\hline IT, & $\varepsilon, 7$ & rq, & 110,0 & $V, r$ & 1,1 & $0 ., 1$ & $17, \xi$. & $r \ldots r$ \\
\hline$r \cdot \varepsilon, \leqslant \wedge$ & $\varepsilon, \wedge$ & $\varepsilon Y, T$ & rq $q, 9$ & $\Lambda, \wedge$ & $r, \Lambda$ & $07, \xi$ & YI,1. & $r \ldots \varepsilon$ \\
\hline Yฯะ,VI & $7, \cdot r$ & $\varepsilon r, q$ & $r \leqslant q, v)$ & 9,9 & $\varepsilon, 1$ & $70,$. & ץ., . & $r \ldots o$ \\
\hline$\varepsilon \cdot 9, \Gamma)$ & 7,1 & $7 V, 1$ & YVT,IA & 9,5 & 0,1 & $\vee q, v$ & $\leqslant \vee, 7$. & $r \ldots r$ \\
\hline$\varepsilon 70,1 V$ & $7,+1$ & $V \vee, \varepsilon$ & To\&,7T & $1 \%, \Lambda$ & $7, Y$ & $11 \leqslant, 7$ & $71,7$. & $r \ldots V$ \\
\hline rVI,.V & $0, V$ & 70,1 & Trו, IT & Ir, 9 & $\varepsilon, q$ & $11 \wedge, r$ & $\vee \wedge, \wedge$. & $r \ldots \Lambda$ \\
\hline $710, \cdot r$ & $0, V$ & $1 \cdot v, 9$ & $r 90, \wedge 9$ & $10, \varepsilon$ & $0, \varepsilon$ & 104,0 & $91, r$. & $r \ldots q$ \\
\hline 19v,.0 & 0,0 & 174,1 & $\varepsilon 0 \cdot, \cdot 1$ & $r \cdot, \Sigma$ & $r, r$ & YAV,V & $1 \leq r, \ldots$ & $r+1$. \\
\hline$\wedge \nmid \wedge, \vee \wedge$ & $0, V$ & $1 \leq 0, \varepsilon$ & $11 \cdot, 0$ & YY, \& & $1 \mathrm{TH}$ & $r \leq q, q$ & $1 \Gamma \varepsilon, \wedge$. & $r+11$ \\
\hline$V \vee \leq, r$ & 0,1 & $1 T M, 0$ & YON, $\Sigma Y$ & rY,r & $1 \wedge, r$ & $r 9 \cdot, 9$ & $1 \leqslant \wedge, 7$. & $r+1 r$ \\
\hline $1 \leq 9 \vee, V$ & 0,97 & rol,r & OrT,91 & $\sum 1, \Gamma$ & $r \cdot, \cdot$ & rVI, E & $1 \wedge \wedge, r \cdot$ & $r .1 r$ \\
\hline$V V \cdot, V$ & $7, \cdot \varepsilon$ & IYV,T & YIT,YY & OY,O & 10,1 & $\sum r r, T$ & $1 \wedge 7, \vee$. & $Y \cdot 1 \leq$ \\
\hline ITYT,O & $7, \leqslant T$ & $r \cdot T, r$ & $r \leqslant \vee, .9$ & $\varepsilon \wedge, 7$ & $19, \mathrm{~V}$ & $\leqslant 00,9$ & $199,1$. & $Y .10$ \\
\hline ヘフォY,O & & $1 \leqslant \vee \wedge, 0$ & $\varepsilon Y \backslash V, r$ & rq9,r & IYY,A & rVVO,I & IrVI,I. & الاجمالى \\
\hline $71 \wedge, \vee 0$ & 0,01 & $1.7, Y 0$ & $r \cdot 1, Y \varepsilon$ & 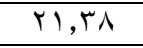 & $\Lambda, \vee V$ & $19 \Lambda, Y Y$ & $9 \vee, 9 \leq$ & المتوسط \\
\hline
\end{tabular}

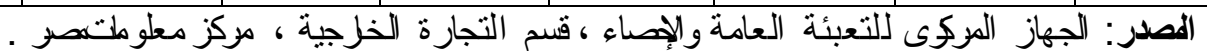

جدول رقم (؛). الإتجاه الزمنسى العام لمؤشرات التجـارة الخارجية لمحصول البطاطس فى مصر خلال

\begin{tabular}{|c|c|c|c|c|c|}
\hline التغير & المثقبط & 广ر & $\mathbf{F}$ & معادلة الإنجاه العام & 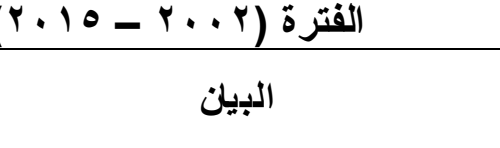 \\
\hline $17, Y \varepsilon$ & १V,१६ & $\cdot, 97$ & **ץ一ा,0 & 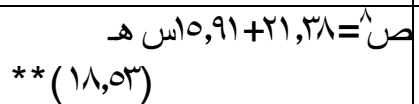 & قيمة لصدرت الكلية ( مليار جنيه) \\
\hline 17,99 & $14, \pi$ & $\cdot, 91$ & ** * & 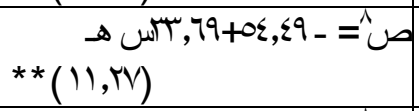 & قيمة الوارلت الكلية ( مليار جنيه) \\
\hline ro,M & $\wedge, \mathrm{W}$ & $\cdot, \mathrm{V} \wedge$ & ** $\leqslant,, \square$ & 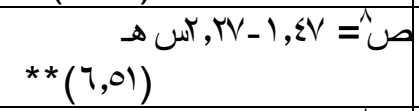 & قيمة لصلدرت الزراعية (مليار جنيه) \\
\hline 泟 & M, « & $\cdot, \wedge \varepsilon$ & ** $ฯ \varepsilon, \mid \varepsilon$ & 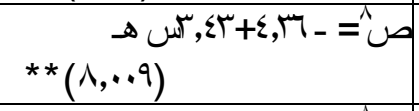 & قيمة الوارلت الزراعية ( مليار جنيه) \\
\hline 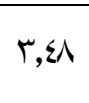 & $\Gamma \cdot 1, \Upsilon \varepsilon$ & $\cdot, 17$ & 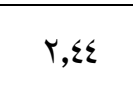 & صد & كميةصدرت البطلس(لفـطن) \\
\hline$r, 19$ & $1.7,10$ & $\cdot, \mathrm{\vee} \wedge$ & 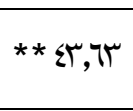 & صـ & قيمةصدرات البطلس(مليون لولاز) \\
\hline I & 0,01 &., 01 & $* * \mid r, 09$ & صـ & متوسطسرصرف الولار(بالجنيه) \\
\hline $1 \leqslant, \lambda$ & 711,10 & $\cdot, \wedge$ & $* * 0,(n)$ & $\begin{array}{l}\text { ص } \\
* *(V, r)\end{array}$ & قيمةصلدرات البطلس (مليون جنيه) \\
\hline
\end{tabular}

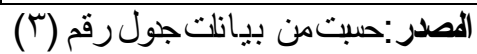

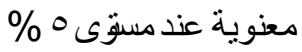


r ـ التركيز الجغرافى للصادرات المصرية من البطاطس لأهم الاول بإستخدام معامل جينى:

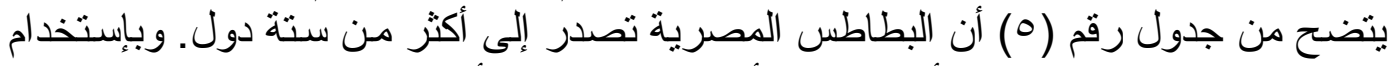

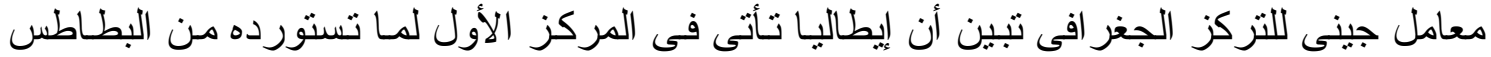

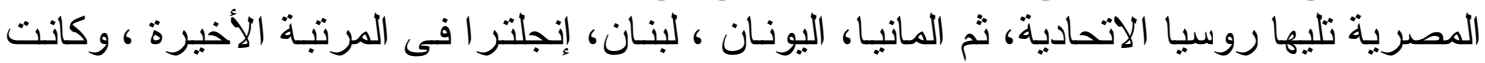

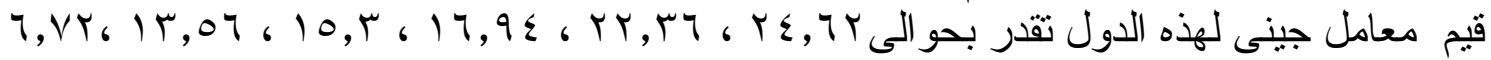

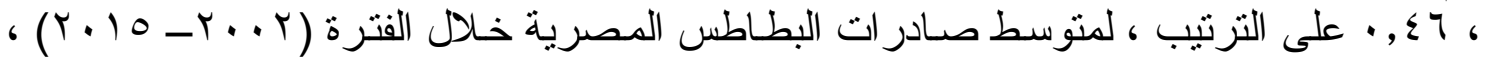

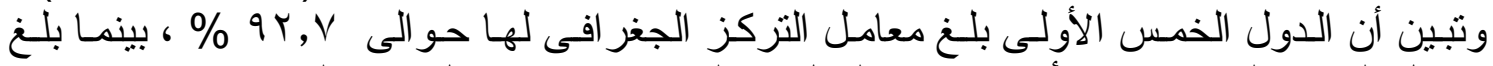

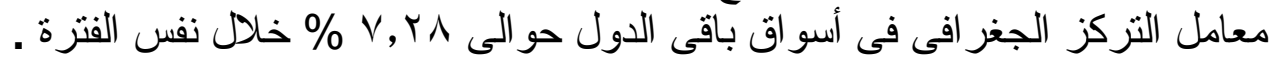

جدول رقم(0). تطور الصادرات المصرية مـن البطاطس إلى دول العـالم وحساب التركيز الجغر افىى

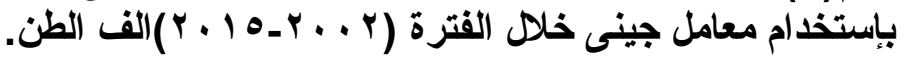

\begin{tabular}{|c|c|c|c|c|c|c|c|c|}
\hline الجملة & اخرى & روسيا & لبنان & الماتيا & انجلترا & اليونان & ايطاليا & السنوات \\
\hline 107,7 & 10,r &.,$\ldots$ & ҮА, T. & 11,09 & $r \leq, 7)$ & $11, \wedge \wedge$ & $0 \leqslant, 01$ & $r \ldots$ \\
\hline 110,0 & $\varepsilon, Y q$ & $\cdot, \cdots 1$ & rr,人т & $\leqslant 0, \wedge 0$ & $r \leqslant, r_{0}$ & $11,7$. & $70,0 \leqslant$ & $r . .1$ \\
\hline Yq\&, . & &., 90 & $\{7,7)$ & $97, V V$ & $1 \mathrm{~V}, 1 \wedge$ & $\{0, \Gamma)$ & $\Lambda V, Y V$ & $r \ldots r$ \\
\hline$r \leq q, V)$ & & $T \varepsilon, \cdot V$ & $7 ., 1$. & rА, Ү. & $M \wedge, Y Y$ & $r 1,1 r$ & $V V, 0$. & $r \ldots r$ \\
\hline$r \vee 7,1 \wedge$ & & $19, \Gamma V$ & $\{7,1$. & $00, \Lambda \varepsilon$ & $10, \Lambda T$ & $\pi \mu, \Sigma \pi$ & $V 0,71$ & $r \ldots \varepsilon$ \\
\hline To\&,77 & & $0 ., 0 \leqslant$ & Or,YT & $\Lambda I, V \mu$ & YY,TT & $\sum 9,0 r$ & $97,9 \leq$ & $r . .0$ \\
\hline r, וTr & & $\vee \cdot, \wedge \wedge$ & Y^, १V & $7 \Lambda, T V$ & $r \cdot,\rangle \wedge$ & $0 ., 9$. & Q1,Yr & $r \ldots r$ \\
\hline $490, \wedge q$ & & $70, .7$ & $\Lambda 0, r V$ & $\varepsilon q, \vee \wedge$ & $r \mu, V I$ & $70, \Gamma \leqslant$ & 1.7, & $r \cdot r$ \\
\hline$\varepsilon 0,, \cdot 1$ & & $1.1,0 \mathrm{~V}$ & $0 \leqslant, 79$ & $\overline{\wedge \Lambda, \wedge r}$ & YY, $\leqslant 0$ & $V \Psi, Y I$ & 1.9, 1. & $r \ldots \Lambda$ \\
\hline $11 \cdot, 0$ & & $r q, \cdot r$ & YY,OY & rq, vo & $I T, \wedge \Lambda$ & $\varepsilon 0, \cdot \varepsilon$ & $r \cdot, r \wedge$ & $r . .9$ \\
\hline$r O \Lambda, \Sigma Y$ & & $7 \Lambda, \Sigma \varepsilon$ & $r r, V I$ & rq,7r & $10, T \varepsilon$ & $0 V, 9 T$ & Tr,TV & $Y .1$. \\
\hline orr,qA & & rrT,qV & $1 V, 7$. & $r 0, V T$ & $r \varepsilon, 19$ & $O V, \Sigma Y$ & $77, \cdot 1$ & $r .11$ \\
\hline$r \mid r, r r$ & & $T Y, 00$ & $Y Y, \wedge V$ & $\varepsilon V, \Gamma_{0}$ & $9, \leqslant 9$ & $r 0,09$ & $\Gamma 0, r V$ & $r \cdot 1 r$ \\
\hline$r \leqslant V, . q$ & & IrV, 9. & $\varepsilon V, V \varepsilon$ & $1 \leqslant, \varepsilon$. & $\Gamma \cdot, 7 \varepsilon$ & $\varepsilon \vee, \Gamma\rceil$ & $\vee 9, .0$ & $r .1 T$ \\
\hline$\varepsilon r \mid V, T_{0}$ & 19,70 & Q & ov1, 9 & $\begin{array}{c}V \backslash \leq, 0 \\
1\end{array}$ & $\begin{array}{c}\text { rAT, } \Sigma \\
1\end{array}$ & $7 \leqslant 0,7$ & I. $\mathrm{A} \wedge, \mathrm{V}$ & \\
\hline & $\cdot, \leqslant 7$ & TY,YT & 11,07 & $17,9 \varepsilon$ & $7, V Y$ & $10, \Gamma$. & $T \varepsilon, T Y$ & \% \% للصادرات \% \\
\hline & ro. & rq. & YI. & rAo & $\varepsilon 7$. & TAV & rvo & سعر الطن/ دولار \\
\hline & $1, \varepsilon$ & $T \vee, 广 \Lambda$ & $\varepsilon \cdot, \wedge 0$ & $01, .4$ & $r \cdot, r \varepsilon$ & $\leq 7,11$ & $v \varepsilon, 19$ & لكميه الصادر سنوي \\
\hline
\end{tabular}

المصدر: الجهاز المركزى للتعبئة العامة والإحصاء ، مركز معلومات قسم التجارة الخارجية ، بيانات غير منشورة.

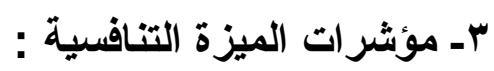

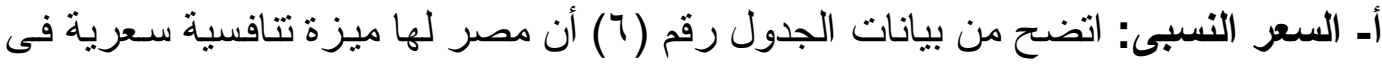

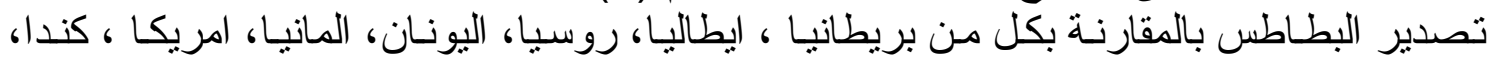

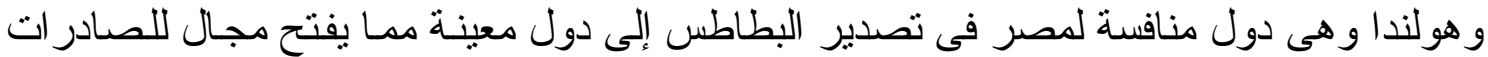

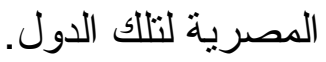


جدول رقم (7). الوضع العالمى وحساب الإتتاج النسبى والسعر النسبى لمصر بين الدول المنافسة

\begin{tabular}{|c|c|c|c|c|c|c|c|}
\hline النسبى * السعر & تصدير الطن & 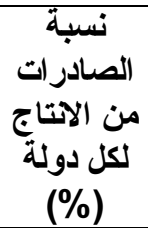 & 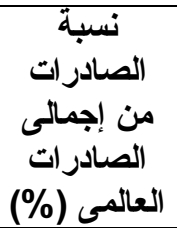 & (الصادرات & الإلى المحلى الانتاج & (الالف طن & أهصدرة الدول \\
\hline$T$ & TOY & $9, \wedge$ & $\varepsilon, \Gamma V$ & $\varepsilon r \mid V, r$ & $\cdot, 91$ & $\varepsilon r V \cdot 1, V$ & مصر \\
\hline $1, r r$ & $\leqslant 70$ & 99,115 & $\cdot, \varepsilon \Gamma$ & $\leqslant 07$ & $\cdot, ., 1$ & 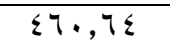 & روسيا \\
\hline $1, r \varepsilon$ & $\sum V_{0}$ & $11, \mathrm{~V}$ & $r, V$ & $r 7.0,0$ & $\cdot, \Sigma \mathrm{V}$ & YYYOQ,T & ايطاليا \\
\hline $1, r \varepsilon$ & $\sum V Y$ & $r, r$ & $\cdot, r 9$ & rVq,A & $\cdot, Y_{0}$ & Ir. $9 r, r$ & اليونان \\
\hline $1, \pi r$ & $\sum 70$ & $1 \Gamma, v$ & YY,O & YITVA & $\Gamma, \Gamma$ & 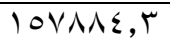 & المانيا \\
\hline $1, \cdot v$ & rᄉ. & $r q, 7$ & 1,70 & 1790,14 &.,$I Y$ & OVI $\{, T$ & لبنان \\
\hline 1,00 & $0 \leq 7$ & $\varepsilon, r$ & $r, v$ & rצro & 1,1 & $\Lambda \leqslant 10$ & بريطانيا \\
\hline $1, \cdot Y$ & 47 & $r \varepsilon, \wedge$ & $T \varepsilon, Y V$ & TMTO. & $r, \cdot$ & 9rNor & هولندا \\
\hline$\cdot, \wedge$ & $Y \wedge \varepsilon, 0$ & $r \wedge, \Lambda$ & ro,o & $r \leqslant \leqslant \wedge$. & 1,09 & ヘะтr. & فرنسا \\
\hline $1,1 \mathrm{~V}$ & $\varepsilon \mid T$, , & Tr,O & $\varepsilon, \wedge \wedge$ & $\leqslant 79$. & $\cdot, \leqslant Y$ & 19110 & امريكا \\
\hline \multirow[t]{3}{*}{1,11} & $r q \varepsilon, \wedge$ & $r v, \cdot \varepsilon$ & $\mathrm{v}, 1 \wedge$ & $79 V$. &., 00 & rovvo & كندا \\
\hline & $r V$. & $\cdot, \cdot \varepsilon$ & $1,9 \mathrm{~V}$ & $191 \mathrm{~V}$ & $\Lambda \Lambda, r$ & EIYIKY. & دول اخرى \\
\hline & & & & $97 . Y V$ & $1 \ldots$ & sTV.VO. & اجمالى \\
\hline
\end{tabular}

الصصدر :شبكة المعلوهت ، قاعدة بيانلت منظمة الاغنية و الزراعة (الفاو ).

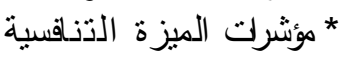

ب- الإتتاج النسبى: اتضح من بيانات الجدول رقم (7) أن نسبة الإنتاج المحلى إلى الإنتاج

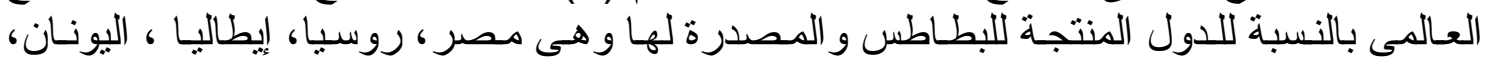

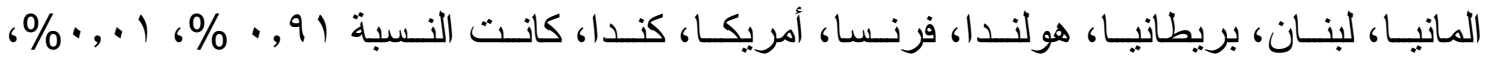

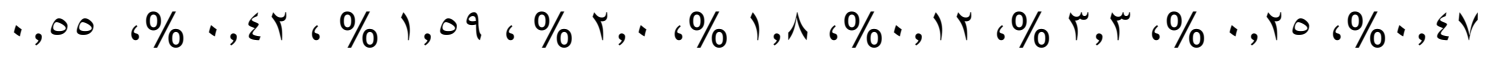

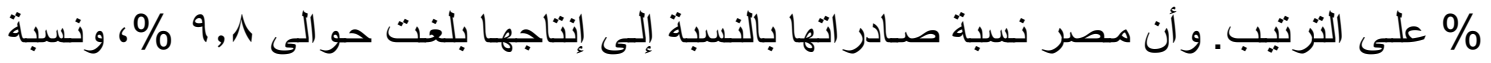

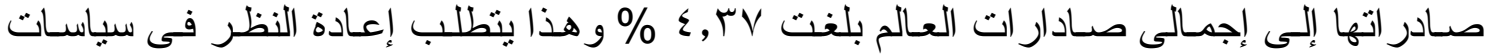
التصدير وتدعيم المركز التنافسى لها. التها.

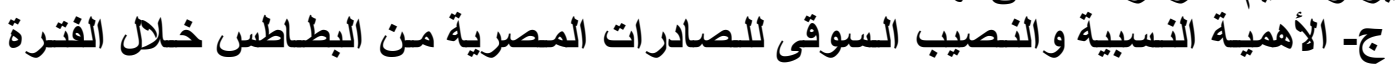

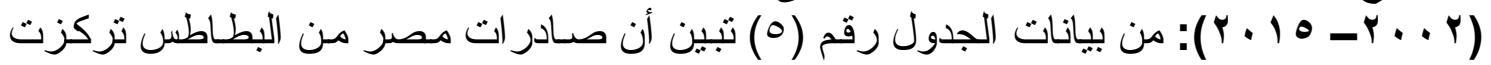

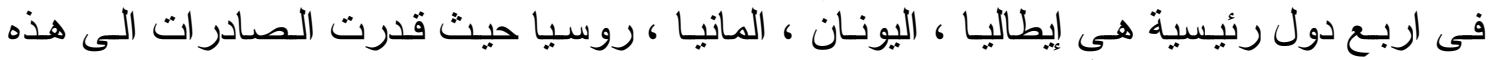

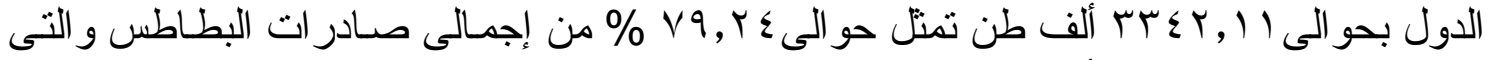

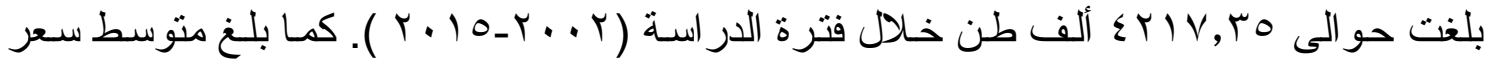

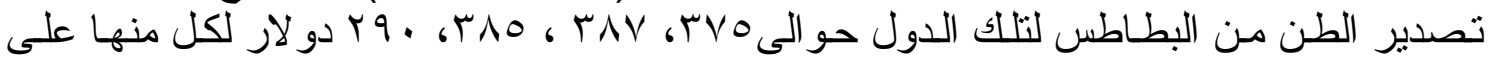

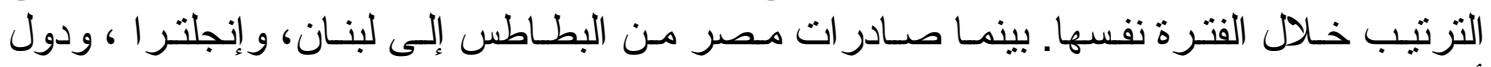

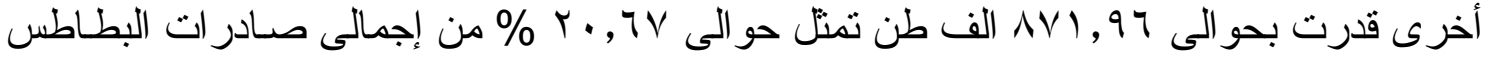

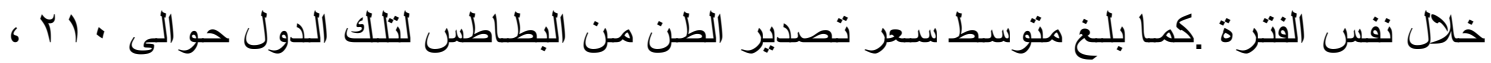

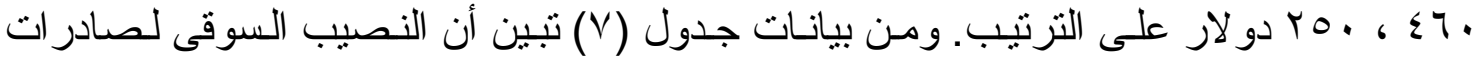

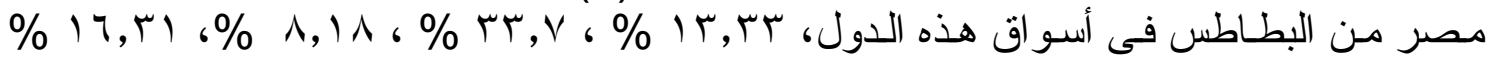

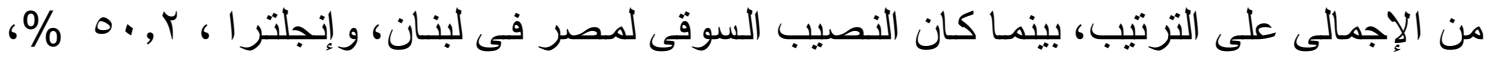

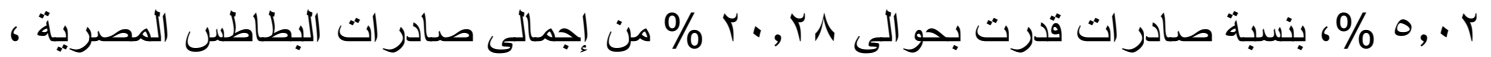

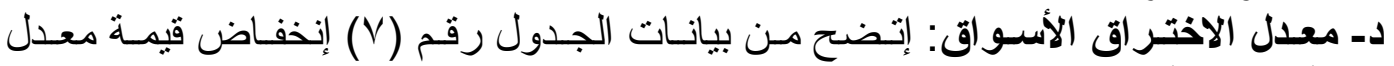

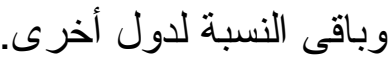

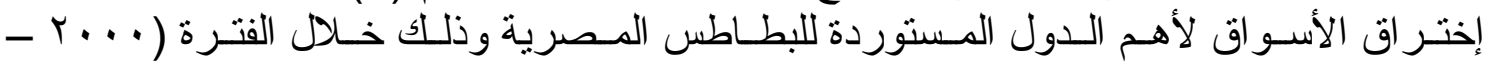




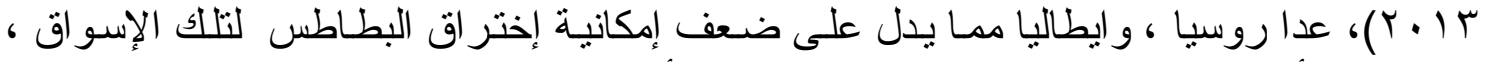

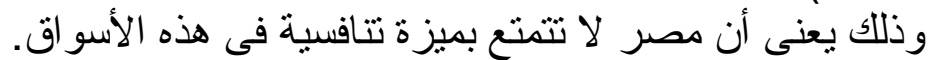

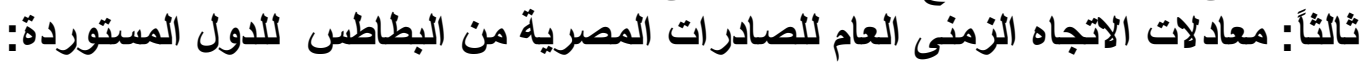

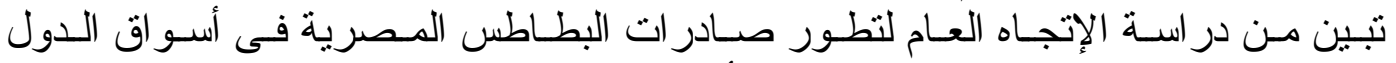

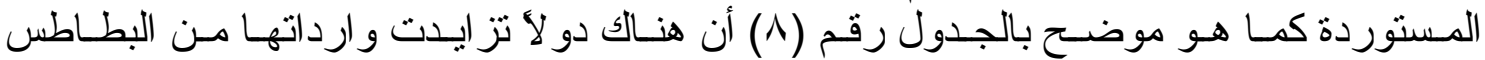

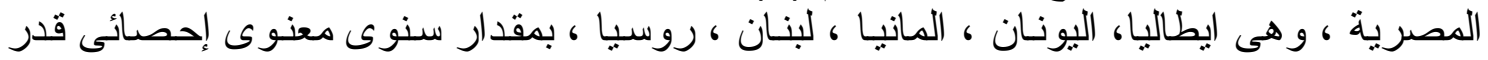

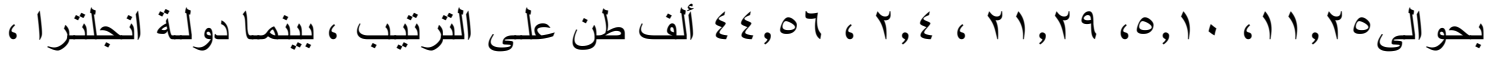

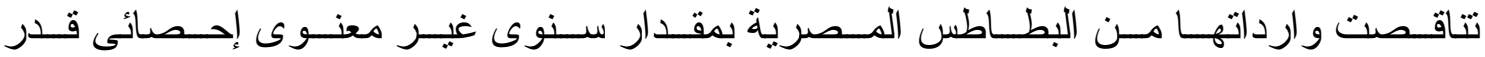
بحو الى 90 , الف طن ود الف ، وذلك خلال فنرة الدر اسة.

جدول رقم (V). إجمالى واردات الاول من البطاطس والنصيب السوقى لمصر فى أسواقها المستوردة

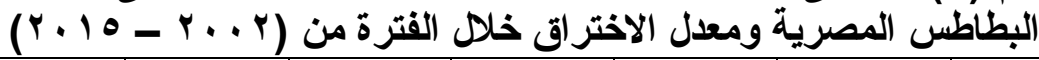

\begin{tabular}{|c|c|c|c|c|c|c|c|c|}
\hline الاجمالى & اخرى دول & روسيا & لبنان & الماتيا & انجلترا & اليونـان & ايطاليا & السنوات \\
\hline & & M & $\varepsilon \varepsilon, \pi$ & 0.111 & $\Sigma) \cdot, r$ & $1 \cdot \varepsilon, 0$ & תוד, 01 & $r \ldots r$ \\
\hline & & $1 \cdots, \mathrm{IV}$ & $0\}, * 1$ & $0 / 9,1$ & $\infty ., 0$ & N,N & $011,(1)$ & $r \ldots r$ \\
\hline & & 194,9 & $V \leqslant, A V$ & ow, 1 & sT., & $11 \leqslant, \varepsilon$ & $\infty$ & $r \ldots \varepsilon$ \\
\hline & & $0.1, \cdot 1$ & $V 7,09$ & 009,0 & $M 9, \varepsilon$ & 100,1 & TYY,90 & $r . .0$ \\
\hline & & $171, Y$ & $W, \leq \varepsilon$ & orर, $+Y$ & זוז & $|\Sigma|, Y$ & & $Y . . T$ \\
\hline & & $M r, o$ & $M, T Y$ & or ,9 & $s<, \varepsilon$ & $11 V, V \varepsilon$ & or, & $r . . V$ \\
\hline & & $M \cdot, q$ & Vo,vz & $T W, \cdot Y$ & M.,1 & $11 \cdot, 9$ & $0,9,77$ & $r \ldots \Lambda$ \\
\hline & & Yol, $Y$ & $11 V, 70$ & $0 r \leqslant, 7$ & $M, 0$ & $1 \leq \cdot, 9$ & or, rar & $r . .9$ \\
\hline & & OV , & $9 T, Y \Lambda$ & \& & $0.9,10$ & IET,r & 099,97 & $r .1$. \\
\hline & & $M \varepsilon, 1$ & $A T, T V$ & ov $\lambda, r$ & Y(17,0) & 170,1 & $7 . \Gamma, Y 0$ & $r .11$ \\
\hline & & Tדז,T & $11 \pi, Y$ & or, & Y乏人, $\varepsilon$ & IT,E & $V \cdot \lambda, V \varepsilon$ & $r \cdot 1 r$ \\
\hline & & $1 \varepsilon 77, Y$ & 99,14 & $701, \varepsilon$ & ru,VA & $I V \varepsilon, T$ & $7 . Y, \circ \wedge$ & $r .1 r$ \\
\hline & & $27,1\}$ & 79,0r & $V \leqslant 7, \cdot \varepsilon$ & $\varepsilon \mid r, 0$ & $1 \% 9,0$ & $7 T V, \cdot Y$ & $r \cdot 1 \varepsilon$ \\
\hline & & E纟,Y & $K, \leq \varepsilon$ & $1.71, r$ & $\pi$ & $191, .0$ & אד,חרד & $r .10$ \\
\hline T.999,А. & & OVAY,O & 11K,A & AMT,E & 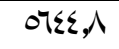 & $1910, \pi$ & War & جملة الواردات \\
\hline \multirow[t]{3}{*}{ סTIV,T } & סק, 19, & . & on, 9 . & $n \leqslant, 01$ & (KN,E. & $7 \leqslant 0,7$. & I. rᄉ, V. & مصر من البطاطس \\
\hline & & $\mid 7, \pi$ & $0 ., r$. & $\Lambda, 1 \Lambda$ & $0,+Y$ & T,V. & سח, & "النصيب السوقى \\
\hline & & $V, W$ & $\cdot, \leqslant 0_{-}$ & Ir,A.- & $r, \cdot 1$ & 1,7 . & $0, r$. & *معدل الإختراق \\
\hline
\end{tabular}

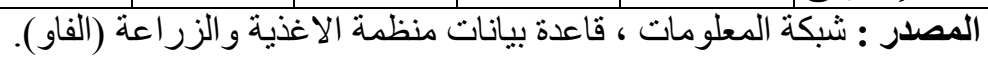
* مؤشرت الميزة التناقسية 
جدول رقم (^). الإجاه الزمنى العام لتطور كمية الصادرات من البطاطس المصرية إلى أهم الدول

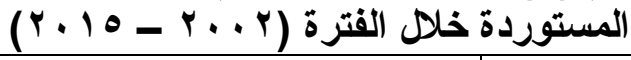

\begin{tabular}{|c|c|c|c|c|c|}
\hline \% التغير & المتوبط & ju & ت & معادلة الإججاه العام & اللول \\
\hline $1, \mathrm{NV}$ & $099, \&$ \& & , 01 & $* * 17,07$ & 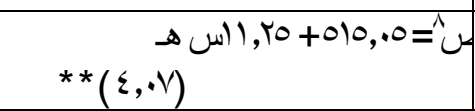 & ريطاليا \\
\hline$r, K$ & $\mathrm{Mr}, \mathrm{W}$ & , 01 & $* * \mid r, \pi$. & 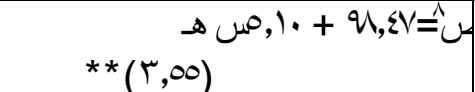 & اليوننا \\
\hline 纳, & גו ג, & ,...0 & ז & 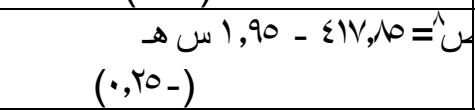 & اتجلترا \\
\hline$\Gamma$, (\$) & נוז, & בq & $* * \gamma, \gamma \wedge$ & 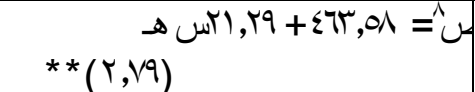 & المانيا \\
\hline$r, 90$ & 1, $1, \% \varepsilon$ & & *ץ,99 & 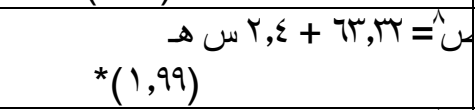 & لبنان \\
\hline $1 ., 99$ & $\{\varepsilon 1,0 V$ & , r & $*_{0, \Omega T}$ & 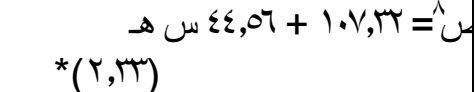 & روسيا \\
\hline
\end{tabular}

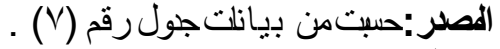
صن': القيمة الثقيرية لكمية البطاطس المصرية س هـ :تثير إلى عنصر الزمن التطن

يعتبر القطاع الزر اعى المصرى من القطاعات ذات الأهمية لتحقيق إنجاز ات عديدة ، يمكن

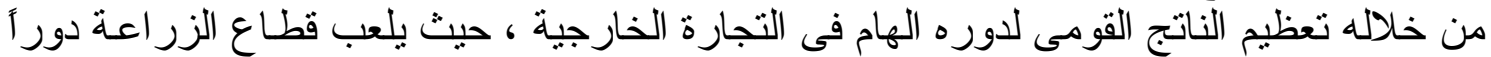

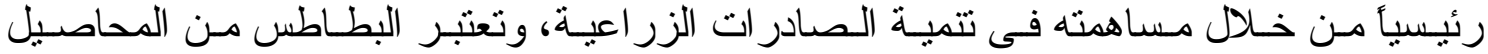

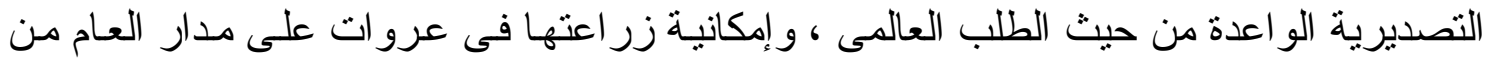

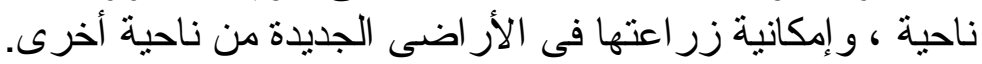

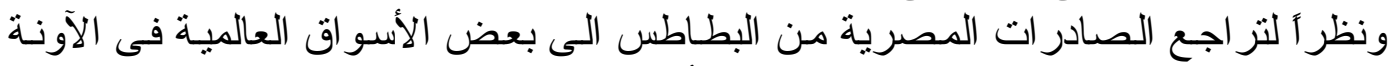

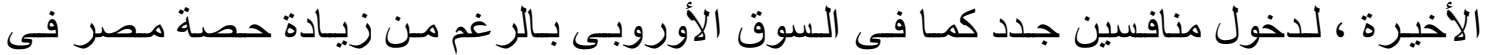

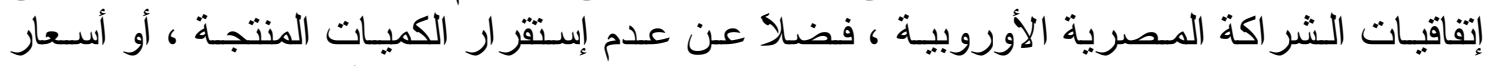

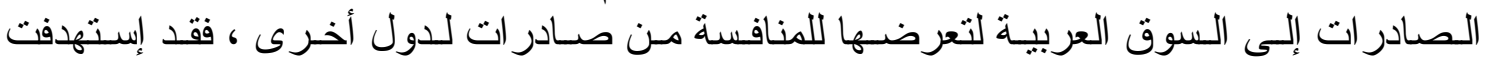

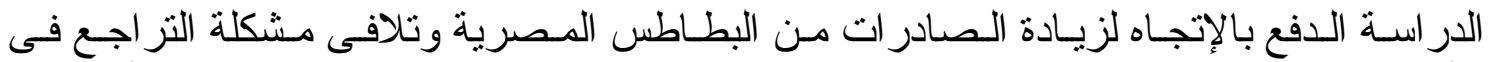

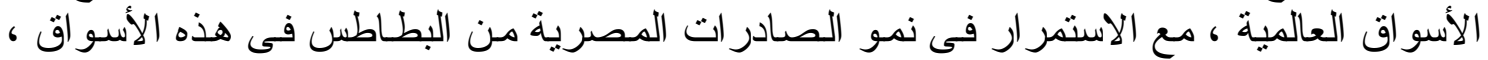

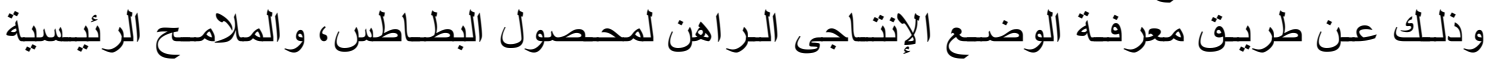

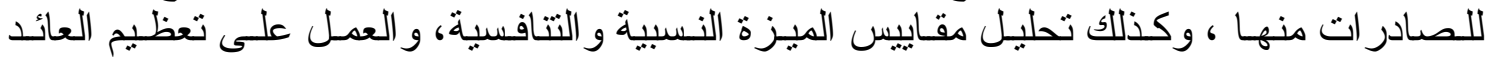

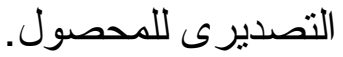
وقد اعتمدت الدر اسـة لتحقيق أهدافها على الأسلوب الوصفي و الكمسي في تحليل البيانـات

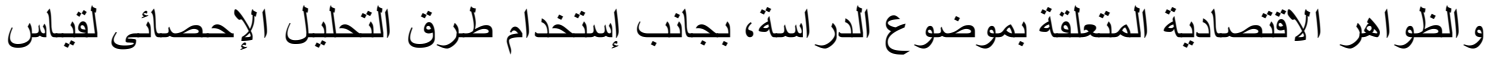

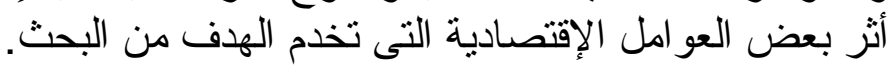

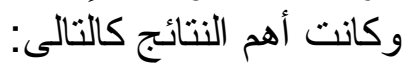

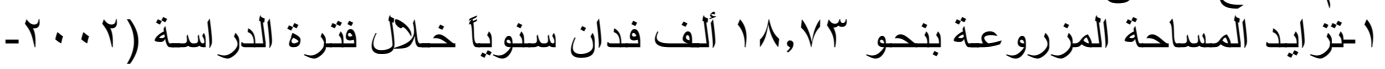

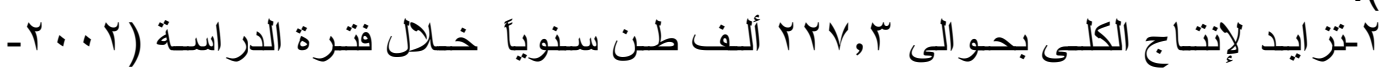




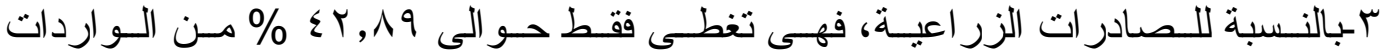

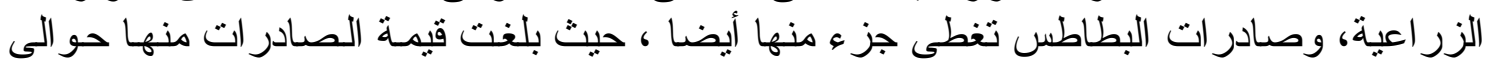

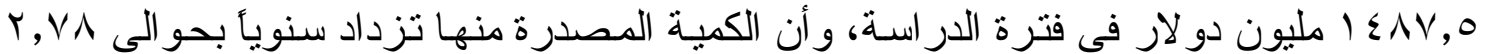

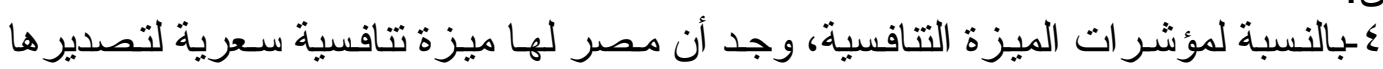
ألف طن.

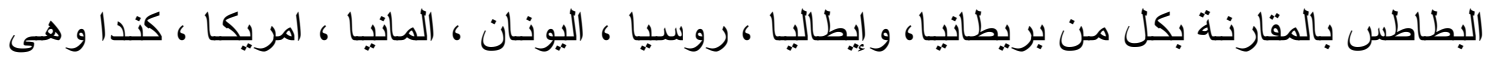

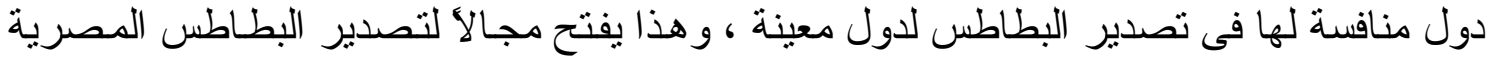
لتلك الدول .

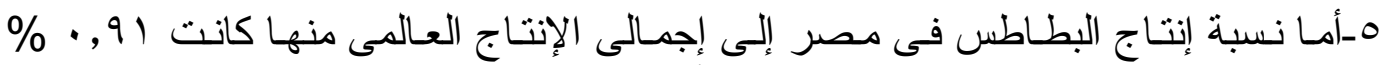

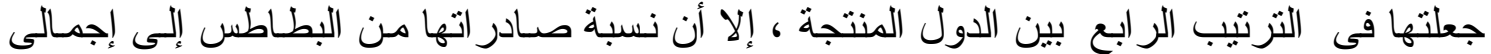

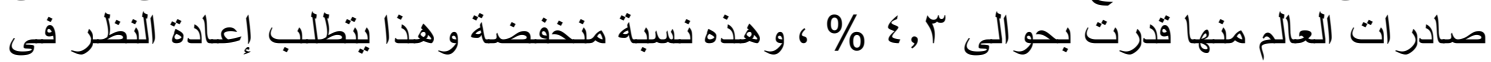
سياسات التصدير وتدعيم المركز التنافسى لها.

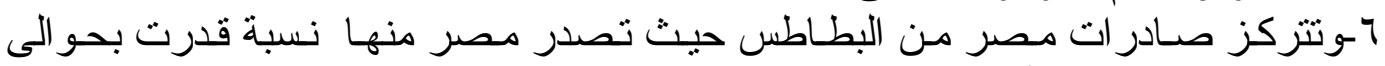

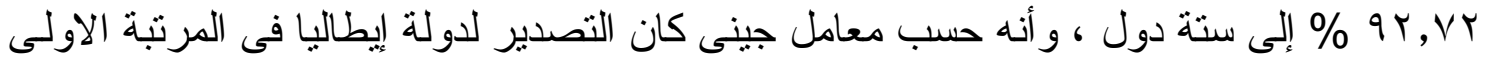

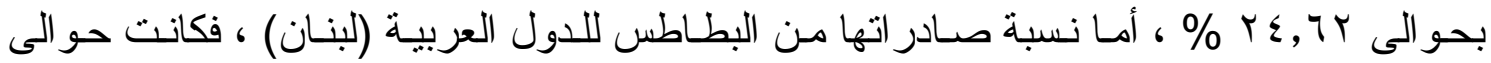

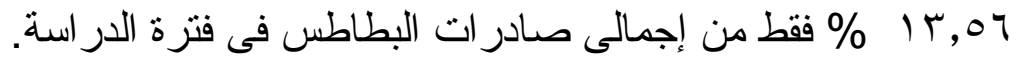

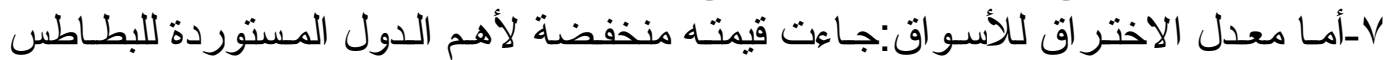

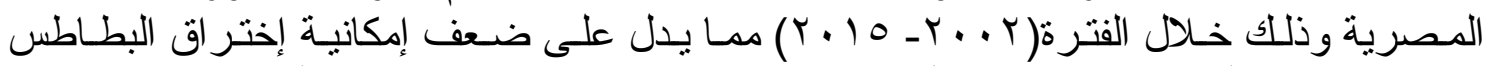

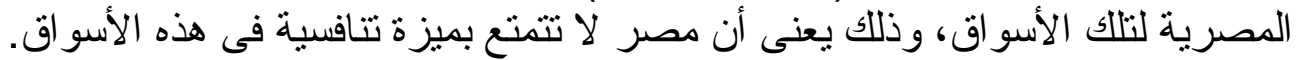

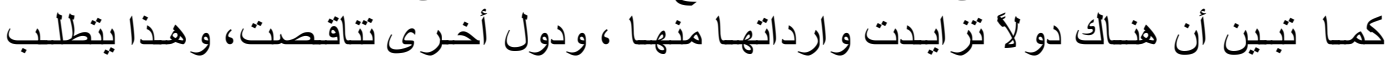

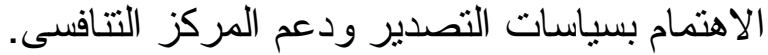

ا ـ الاهتمام بزر اعة البطاطس للتصدير ، وزيادة الحافز للمنتجين و المصدرين ، الأمر الذى

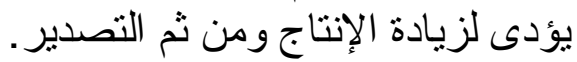

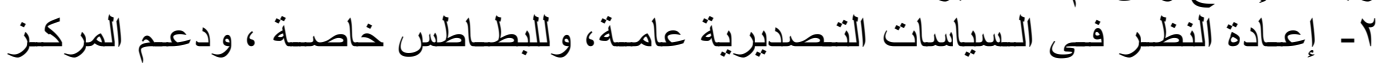

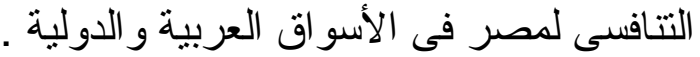

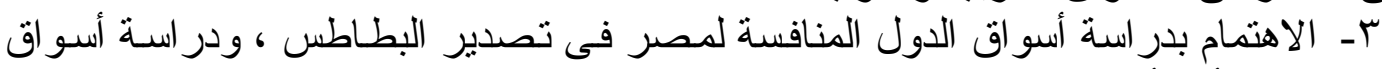

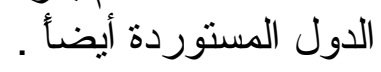

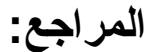
ا - الجهاز المركزى للتعبئة العامة و الإحصاء ، قدم التجارة الخارجية ، القاهرة .

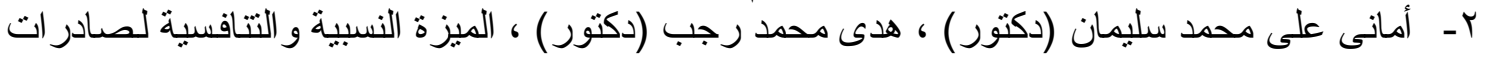

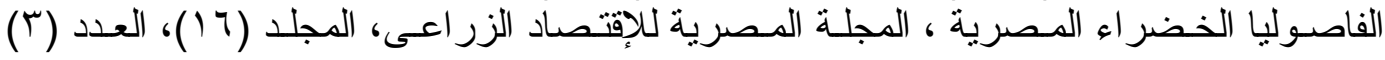

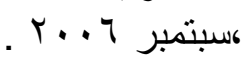

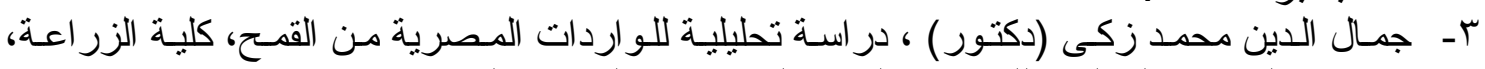

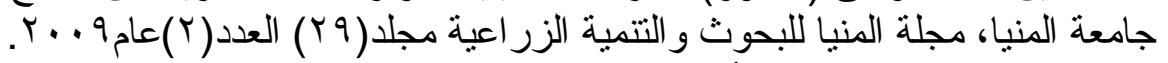

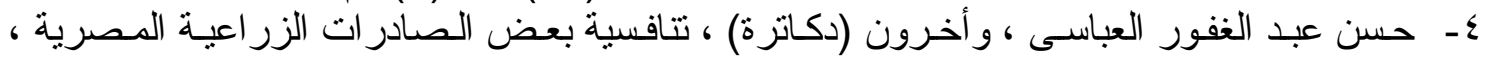

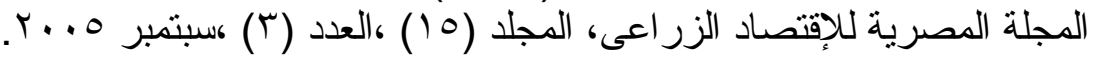

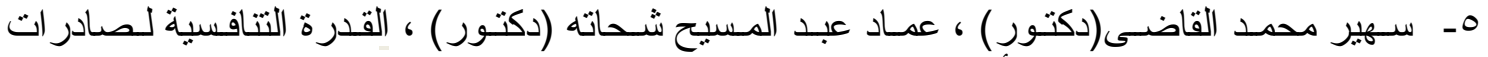

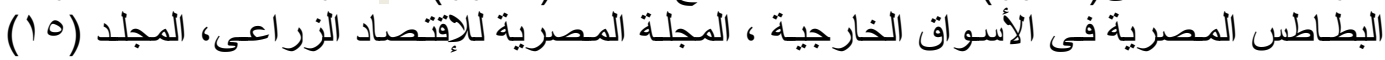

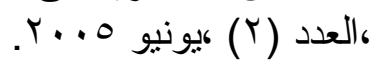

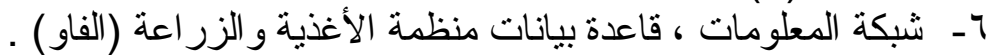
V- شبكة المعلومات، قاعدة بيانات الجهاز المركزى للتعبئة العامة و الإحصاء. 


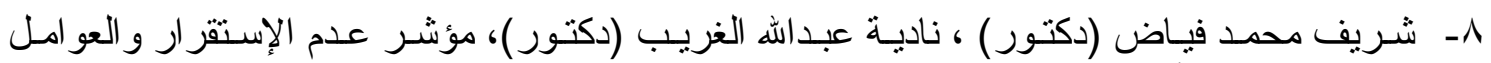

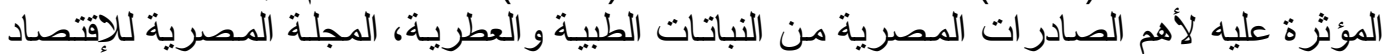

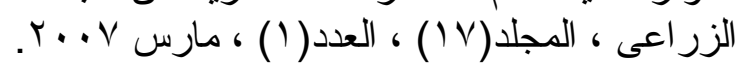

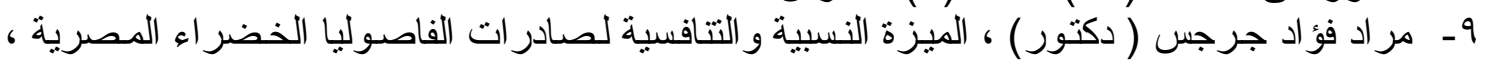

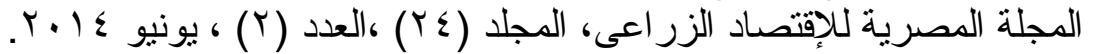

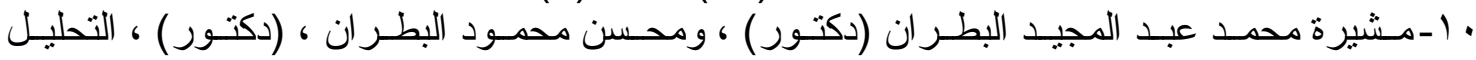

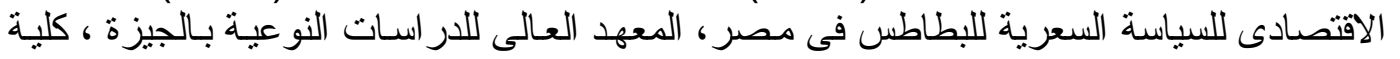

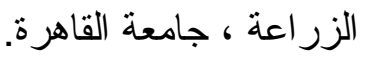

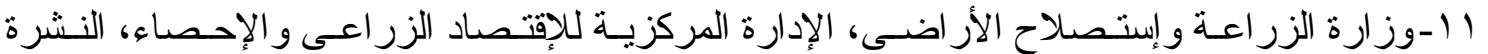
الإقتصادية، أعداد متقرقة. 


\title{
Comparative Advantage and Competitiveness of Egyptian Potato Exports in World Markets
}

\author{
Ahmed AboEL-Maged Hashem ${ }^{1}$; Hatem Mahmoud El-Sanosy ${ }^{2}$ and Morad F. Gerges ${ }^{2}$ \\ ${ }^{1}$ Faculty of Agriculture, Sohag University. \\ ${ }^{2}$ Agric, Econ, Res, Instit- Agric, Res, Center.
}

\section{Summary}

The Egyptian agricultural sector is considered one of the important sectors to achieve many achievements, through which the national product can be magnified for its important role in foreign trade. The agricultural sector plays a major role through its contribution to the development of agricultural exports. Potato is one of the most promising export crops in terms of global demand, and the possibility of cultivating them in new lands on the other hand.

In view of the decline of Egyptian exports of potatoes to some international markets in recent times, to enter new competitors as in the European market despite the increase of Egypt's share in the Egyptian-European partnership agreements, as well as instability of the quantities produced, or export prices to the Arab market for competition from exports to countries Other, The study aimed at boosting the export of Egyptian potatoes and avoiding the problem of the decline in international markets, while continuing to grow Egyptian exports of potatoes in these markets by knowing the current production status of potato crop and the main features of exports. And work to maximize the export yield of the crop.

The study was adopted to achieve its objectives on the descriptive and quantitative method in analyzing the economic data and phenomena related to the subject of the study, as well as the use of statistical analysis methods to measure the effect of some economic factors that serve the objective of the research. The most important results were as follows:

1- The cultivated area increased by 18.73 thousand feddans annually during the study period (2002-2015).

2- An increase of kidney production by 227.3 thousand tons per year during the study period (2002-2015).

3. For agricultural exports, it covers only about $42.89 \%$ of agricultural imports. Potato exports also cover part of the exports, with exports amounting to about US \$ 1,487.5 million in the period of study. The exported quantity increases annually by 2.78 thousand tons.

4 - For the indicators of competitive advantage, it was found that Egypt has a competitive advantage for the export of potatoes compared to Britain, Italy, Russia, Greece, Germany, America, Canada, which are competing countries in the export of potatoes to certain countries.

5. The percentage of potatoes production in Egypt to the total world production was $0.91 \%$, which made it the fourth largest among the producing countries. However, the proportion of potato exports to the total exports of the world was estimated at $4.3 \%$. This is a low ratio. This requires reconsideration of export policies. Competitive position. 
6- The Egyptian exports of potatoes are concentrated in Egypt, where $92.72 \%$ of them are exported to six countries. According to the Gini coefficient, exports to Italy ranked first with about $24.62 \%$. The share of potato exports to Arab countries (Lebanon) was about $13.56 \%$ Of total potatos exports in the study period.

7- The market penetration rate was low for the Egyptian potato importers during the period (2002-2015), which indicates that Egypt's potato can not penetrate these markets. This means that Egypt does not have a competitive advantage in these markets.

It has also been shown that there are countries whose imports have increased and others have decreased. This requires attention to export policies and support for the competitive position.

\section{Recommendations:}

1- Interest in the cultivation of potatoes for export, and to increase the incentive for producers and exporters, which leads to increased production and then export.

2- Reconsider the general export policies, and especially potatoes, and support the competitive position of Egypt in the Arab and international markets.

3- Interest of study the markets of Egypt's competitive countries in the export of potatoes and to study the markets of importing countries as well. 